\title{
Metodología para la comparación de sistemas de planificación de recursos empresariales para servicios logísticos portuarios
}

\author{
Methodology for comparison of Enterprise Resource Planning \\ systems for port logistics \\ Paola A. Sánchez-Sánchez ${ }^{1 *} \quad$ José Rafael García-González ${ }^{2} \quad$ Luis Eduardo Ortiz-Ospino ${ }^{3}$ \\ Recibido 1 de diciembre de 2015, aceptado 16 de septiembre de 2016 \\ Received: December 1, 2015 Accepted: September 16, 2016
}

\begin{abstract}
RESUMEN
La implementación de un sistema de planificación de recursos empresariales (ERP - Enterprise Resource Planning), a menudo resulta ser un proceso complicado, que consume muchos esfuerzos y recursos. Una fase crítica en la implementación es la selección de los módulos que debe tener el sistema, toda vez que una inadecuada elección puede resultar en el fracaso del proceso. En la literatura existen metodologías orientadas a la selección de sistemas ERP de forma general, y no a la comparación particular de los elementos de diferentes sistemas. En este artículo se desarrolla una metodología de comparación de ERP para logística portuaria, cuya finalidad es la adecuada elección de los módulos que debe contener el sistema. La validación se realizó mediante la comparación de tres ERP para logística portuaria, resultando en la especificación de un sistema con doce módulos que permitirían cumplir los requerimientos de cualquier sistema de este tipo.
\end{abstract}

Palabras clave: Sistemas de planificación de recursos empresariales para logística portuaria, metodologías de comparación de plataformas de planeación de recursos, métricas de comparación de software.

\begin{abstract}
Implementing an Enterprise Resource Planning system often proves to be a complicated process, which consumes a lot of efforts and resources. A critical step in the implementation is the selection of the modules that the must have, since an inadequate choice may result in failure of the process. In literature, there are oriented methodologies towards general ERP selection, and particularly not to compare the elements of different systems. This article presents a methodology for comparing port logistics ERP, whose purpose is the right choice of modules must contain the system develops. The validation was performed by comparing three ERP for port logistics, resulting in the specification of a system with twelve modules that would allow to meet the requirements of any system of this category.
\end{abstract}

Keywords: Enterprise Resource Planning for port logistics, Enterprise Resource Planning comparison methodologies, metrics software comparison.

1 Grupo de Investigación Ingebiocaribe. Universidad Simón Bolívar. Barranquilla, Colombia.

E-mail: psanchez9@unisimonbolivar.edu.co

2 Grupo de Investigación Educación, Ciencias Sociales y Humanas. Universidad Simón Bolívar, Barranquilla, Colombia. E-mail: jgarcia122@unisimonbolivar.edu.co

3 Facultad de Ingeniería. Universidad Simón Bolivar. Barranquilla, Colombia. orcid.org/0000-0002-9334-4026.

E-mail: lortiz27@unisimonbolivar.edu.co.

* Autor de correspondencia 


\section{INTRODUCCIÓN}

Un sistema para la planificación de recursos empresariales (ERP - Enterprise Resource Planning), se refiere a una herramienta computacional diseñada para gestionar, de forma integral, la información procedente del flujo de material, personal y recursos financieros de una organización, en una base de datos común [1]. Un ERP, típicamente, gestiona procesos como la producción, logística, distribución, inventario, envíos, facturas, clientes, recursos humanos, calidad y la contabilidad de la empresa.

Las pequeñas y medianas compañías que incursionan en la necesidad de contar con un sistema de gestión ERP, a menudo, se enfrentan a la problemática de i) contratar un servicio especializado, ii) contratar un sistema general, o iii) desarrollar un sistema propio. La opción de contratar un servicio especializado, es decir, que una compañía desarrolle y gestione por ellos el sistema, generalmente, no es la mejor elección debido a los altos costos que esto genera y el temor de ceder autonomía en el manejo de la información [2].

Ahora bien, las prácticas más comunes son la adopción de un ERP general, que es posteriormente adaptado a sus requerimientos específicos, así como el desarrollo de un sistema a la medida. Bien sea que se elija la opción de un ERP general o que se quiera desarrollar un sistema propio, la organización se debe enfrentar al dilema de decidir que módulos incluir.

La implementación exitosa de un ERP implica, por tanto, una apropiada selección de los módulos que lo componen, de tal forma que estos se adecuen a los requerimientos de la organización [3]. Diversos autores han documentado los beneficios e implicaciones que para una organización puede conllevar una selección inadecuada del sistema ERP [4-8].

Las metodologías usadas tradicionalmente para la selección de ERP se basan en la puntuación, ranking, análisis jerárquicos, modelos de programación no lineal y optimización matemática, análisis multicriterio, entre otros. Sin embargo, las limitantes de estos tipos de metodologías radican en la simplificación de los análisis que, a menudo, conduce solo a dirigirse a factores financieros como costos y beneficios, y evaluaciones centralizadas en niveles altos de la organización, desconociendo la evaluación de criterios funcionales.

Ahora bien, la selección específica de los módulos que componen el sistema ERP puede hacerse basado en un análisis comparativo de diferentes sistemas ya existentes, no obstante, en la literatura no existe una metodología que oriente dicha comparación, especialmente, de luces para visionar los criterios adecuados para hacerlo.

En sistemas de logística portuaria la selección de los módulos es difícil, en la medida que son muchos los agentes que intervienen en el sistema, resultando en una cantidad de variables y un volumen de información considerable [9].

El objetivo de este artículo es proponer una metodología orientada a la comparación de sistemas ERP para logística portuaria, como mecanismo que permita determinar cuáles módulos deben ser incluidos en un sistema de este tipo. Para esto, en la sección dos, se discute brevemente el estado del arte en metodología de selección de sistemas ERP; la metodología propuesta para la comparación de sistemas ERP de logística portuaria es presentada en la sección tres; los resultados de la aplicación de la metodología propuesta a tres sistemas ERP se presentan en la sección cuatro; finalmente, en la sección cinco se exponen las principales conclusiones obtenidas.

\section{METODOLOGÍAS PARA LA SELECCIÓN DE SISTEMAS ERP}

Desde hace ya algunos años, los sistemas de información tipo ERP han ido tomando gran importancia en los procesos de negocios de las organizaciones. Según [10], estos sistemas son paquetes que se configuran para integrar información y procesos de las áreas funcionales de una organización [véase, también, [11-12]].

La implementación de un ERP, típicamente, es realizada como un proyecto que implica varias fases, entre ellas la más crítica corresponde a la selección del sistema que mejor se adecua a las necesidades de la organización, toda vez que, una falla en esta fase puede conducir al fracaso del proyecto [13-14].

El proceso de selección de un ERP es considerado una tarea difícil, que consume muchos recursos 
en tiempo y costo; principalmente, debido a la dificultad de establecer los requerimientos de la organización, la complejidad de los paquetes ERP disponibles, y las diversas alternativas de sistemas ERP en el mercado [7]. En la práctica, el proceso de selección de ERP implica la evaluación de varios factores, entre ellos la funcionalidad del sistema con base en los requerimientos de la organización.

Tal como lo afirma [15], un factor primordial en la selección de un ERP es el nivel de ajuste con los objetivos de negocio de la organización; no obstante, [16] señalan aspectos como el costo, el grado de conocimiento de los proveedores, etc.; [17-19] resaltan, además, las competencias tecnológicas de los empleados, el tamaño de la organización y la compatibilidad con sistemas ERP.

En la literatura se han propuesto diferentes metodologías orientadas a la selección de un sistema ERP. Como ejemplo de estas metodologías encontramos:

- [20] desarrollan un modelo basado en un proceso analítico de red (ANP - Analytic Network Process) como herramienta para la toma de decisiones acerca del problema de selección de ERP.

- El método propuesto por [21] para el proceso de selección de ERP se basa en un proceso de análisis jerárquico (AHP-Analytic Hierarchy Process) que permite evaluar los atributos más apropiados para cada organización (véase también [22]).

- [23] presentan un modelo para la adquisición de un sistema ERP basado en la evaluación comparativa de diferentes sistemas, el que consiste de seis etapas: planeación, búsqueda de información, selección, evaluación, elección, y negociación.

- [7] desarrollan una metodología que considera tanto criterios objetivos como subjetivos para la selección de un ERP.

- [6] propone un marco basado en el Nominal Group Technique (NGT) y AHP para seleccionar un sistema ERP.

- [24] desarrollan un modelo de toma de decisiones multicriterio.

- [25] presentan un modelo de plan estratégico para la evaluación y selección de un sistema ERP que toma en cuenta los tres niveles de una organización: estratégico, técnico y ejecutivo.
- [26] presentan una metodología basada en dos pasos: identificación de las propiedades de sistemas ERP existentes, y el uso de un modelo matemático para minimizar el costo de contratación e integración.

- [27] desarrollan una metodología híbrida basada en una identificación de criterios, un análisis jerárquico y un ranking basado en preferencias que permiten seleccionar un ERP.

- En un trabajo posterior [28] desarrollan un método para seleccionar el mejor sistema ERP usando una combinación de ANP y PROMETHEE (Preference Ranking Organization Method for Enrichment Evaluations)

Las diferentes metodologías expuestas orientan criterios para la selección y/o adquisición de sistemas ERP, estas se basan en determinar, bien sea, las necesidades de la organización o en evaluar el grado de ajuste de diferentes sistemas ya existentes. Por lo tanto, no se tiene en cuenta la comparación funcional de los módulos que componen los sistemas.

\section{METODOLOGÍA PROPUESTA PARA LA COMPARACIÓN DE SISTEMAS ERP ORIENTADOS A LOGÍSTICA PORTUARIA}

La estrategia que se propone se basa en la comparación funcional de diferentes sistemas ERP existentes con el fin de establecer los módulos adecuados que debe contener una herramienta orientada a logística portuaria. Esta metodología intenta organizar el proceso de selección de un ERP para que la empresa pueda escoger el sistema que mejor cumpla con sus requisitos basándose en cuestiones que no sean solo económicas.

La estrategia que se propone busca establecer parámetros de comparación que permitan establecer los módulos deseables para un sistema ERP de logística portuaria, evaluando alternativas del mercado en aspectos funcionales y técnicos, de los módulos que componen el sistema, y no en aspectos económicos, ofreciendo pautas para la puesta en marcha del proyecto de implementación del ERP. La metodología no provee herramientas para definir si un sistema ERP es mejor que otro o no, toda vez que el objetivo es ayudar a establecer los módulos que deben ser seleccionados para el diseño e implementación de un ERP portuario. 
La metodología propuesta para la comparación de sistemas ERP orientados a logística portuaria se basa en los siguientes pasos:

Paso 1. Búsqueda de herramientas ERP para logística portuaria.

Paso 2. Definición de métricas de comparación de herramientas.

Paso 3. Comparación de herramientas.

Paso 4. Selección de módulos.

La Figura 1 muestra un diagrama de flujo para la comparación de sistemas ERP; los detalles de cada paso se presentan a continuación.
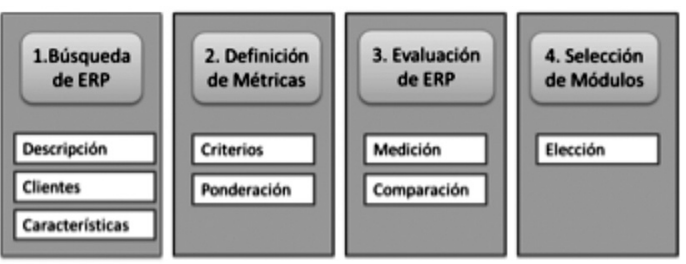

Figura 1. Esquema de comparación de ERP.

\section{Búsqueda de herramientas ERP para logística portuaria}

La fase de búsqueda de herramientas ERP está orientada al análisis exploratorio de diferentes sistemas tipo ERP existentes orientados a logística portuaria con el fin de identificar aspectos relacionados con el ambiente en el que se desarrolla, su funcionalidad, clientes que lo contratan y características.

Dicha búsqueda puede definirse como un proceso de aproximación a los sistemas disponibles mediante un análisis subjetivo, con el fin de elegir aquellas herramientas que ofrezcan tanto elementos generales, como funcionalidades específicas.

\section{Descripción del ERP}

Cada sistema ERP posee aspectos particulares que permiten establecer diferencias entre otros sistemas. Dichos aspectos están relacionados con su rango de aplicación y funcionalidad general.

Rango de aplicación: La búsqueda inicial de sistemas ERP gira en torno al rango de aplicación, es decir, el objeto de negocio de la organización; en nuestro caso particular, sistemas ERP orientados a logística portuaria.
Funcionalidad general: La descripción general del ERP está orientada a identificar las características funcionales del sistema que permiten establecer, tanto similitudes como diferencias.

\section{Clientes del ERP}

El análisis exploratorio inicial requiere establecer la cobertura territorial y los clientes de cada sistema ERP; esto, igualmente, con el fin de establecer la globalidad funcional del sistema.

Cobertura territorial: La cobertura territorial se asocia a la región donde tiene mayor influencia el sistema ERP. El dominio de un sistema puede ser mundial; sin embargo, es necesario establecer el nivel de respaldo que en el territorio de ejecución de la organización tiene el sistema.

Clientes: La búsqueda de clientes de un sistema ERP se refiere a las organizaciones que contratan un servicio especializado. El número de clientes, y el tamaño de estos, permiten dar una idea de la confiabilidad del sistema.

\section{Características del ERP}

La búsqueda de herramientas debe también girar en torno al análisis de las características estructurales de cada ERP; esto es, establecer la forma como está organizado cada sistema. Más allá de determinar que hace (características funcionales), se requiere identificar cómo se organiza para hacerlo (características estructurales).

Este proceso consiste en el análisis y comparación de las características generales de cada uno de los sistemas seleccionados.

\section{Definición de métricas de comparación de herramientas}

La búsqueda e identificación de cada herramienta, debe basarse en aspectos funcionales, no funcionales y en otras características fundamentales de desempeño y desarrollo, por lo que se hace necesario establecer métricas de comparación entre las diferentes herramientas.

En esta etapa se pretende definir las métricas de comparación de los sistemas ERP, las que enmarcan los criterios que serán utilizados para realizar la comparaciónentre los diferentes sistemas ERP; para esto, se deben tener en cuenta las diferentes necesidades que se puedan presentar. Asimismo, se debe establecer el nivel de ponderación que pueda tener cada criterio. 


\section{Criterios}

Las métricas o criterios de comparación se refieren a aquellos elementos que permiten valorar el grado de cumplimiento de un factor o característica.

Las métricas brindan la posibilidad de medir todo aquello que se expresa como una herramienta numérica. La Figura 2 expresa el esquema de medición propuesto por MacCall para medir las bondades de una herramienta de software basado en sus características funcionales [29].

Para la comparación de sistemas ERP la evaluación se basa en del desempeño, según los siguientes elementos: Comunicación de datos, Procesamiento distribuido, entrada on-line de datos, Diseño para la eficiencia de usuario final, Actualización on-line, Puestos múltiples, Facilidad de cambio.

Comunicación de datos: Este factor permite determinar el nivel de transmisión de información que tiene una determinada herramienta. Determina lo accesible de la información y el grado de interactividad.

Procesamiento distribuido: Hace referencia a que los componentes del sistema están distribuidos en servidores o procesadores diferentes. Permite dar una idea acerca de la efectividad, accesibilidad y complejidad del sistema.
Entrada on-line de datos: Bajo este criterio se cuantifica la entrada de datos en línea prevista para el sistema.

Diseño para la eficiencia de usuario final: Esta métrica hace énfasis en la amigabilidad del sistema y su facilidad de uso, buscando aumentar la eficiencia del usuario final. Se orienta a evaluar aspectos funcionales como la ayuda para la navegación, menús, documentación y ayuda on-line, movimiento automático del cursor, scrolling vertical y horizontal, impresión remota, soporte multilingüe, entre otros.

Actualización on-line: Es una medida de las posibilidades de actualización en línea de los archivos lógicos internos que tiene el sistema.

Puestos múltiples: Esta medida hace referencia a si el sistema ha sido específicamente proyectado, diseñado y mantenido para ser instalado en múltiples equipos de una organización o para múltiples organizaciones.

Facilidad de cambio: Mide si el sistema ha sido específicamente proyectado y diseñado mediante módulos o vistas, que faciliten su mantenimiento. Este criterio se orienta a evaluar aspectos funcionales como las facilidades de consultas e informes flexibles para atender necesidades simples, medias o complejas y la efectividad en los cambios.

\section{Use $\quad$ Factor $\quad$ Criteria}

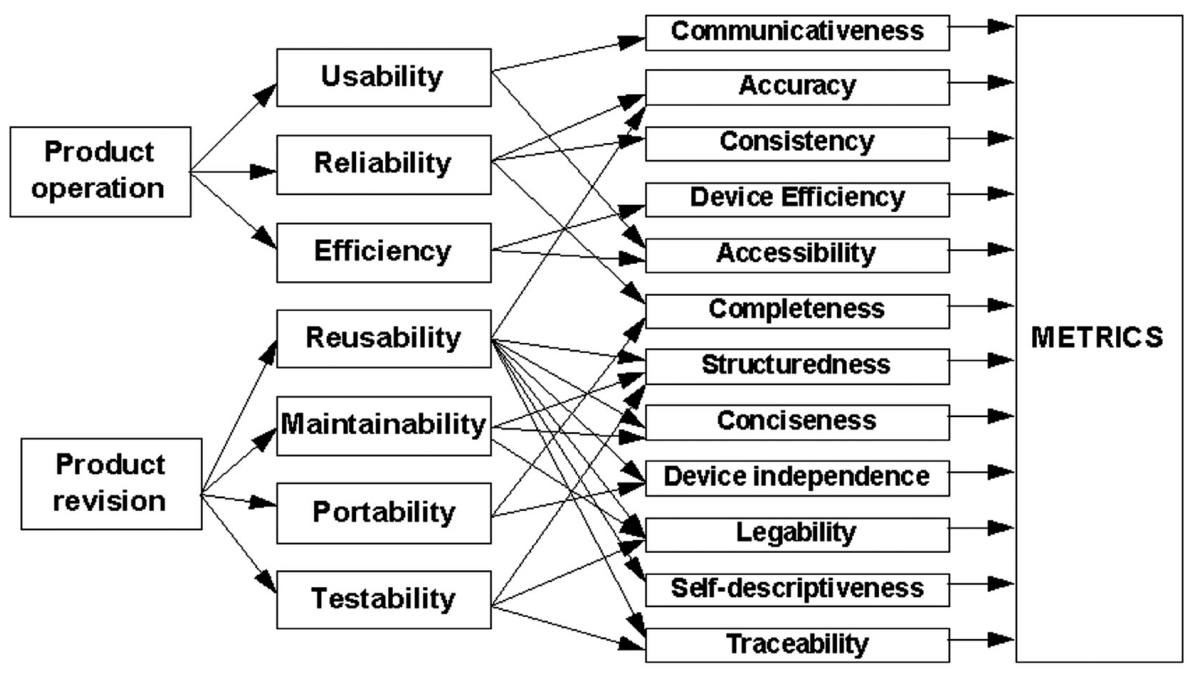

Figura 2. Esquema de métricas propuesto por MacCall. 


\section{Ponderación}

La comparación de ERP según los criterios enunciados requiere definir el valor de medición de cada factor. Para facilitar la medición se han asignado puntuaciones de cero (0) a cinco (5), según el nivel de cumplimiento [Véanse Tablas 1-7].

Tabla 1. Ponderación de comunicación de datos.

\begin{tabular}{|c|l|}
\hline \multicolumn{2}{|c|}{ Comunicación de datos } \\
\hline Grado & \multicolumn{1}{|c|}{ Descripción } \\
\hline 0 & El sistema es aislado del exterior. \\
\hline 1 & $\begin{array}{l}\text { El sistema ofrece entrada de datos remota o } \\
\text { con utilización de periféricos de salida remotos } \\
\text { exclusiva. }\end{array}$ \\
\hline 2 & $\begin{array}{l}\text { El sistema ofrece entrada de datos remota y con } \\
\text { utilización de periféricos de salida remotos. }\end{array}$ \\
\hline 3 & $\begin{array}{l}\text { El sistema ofrece una captura de datos en } \\
\text { línea o posee un sistema de teleproceso que } \\
\text { transfiere los datos. }\end{array}$ \\
\hline 4 & $\begin{array}{l}\text { El sistema ofrece varios teleprocesos pero con } \\
\text { el mismo protocolo de comunicación. }\end{array}$ \\
\hline 5 & $\begin{array}{l}\text { El sistema ofrece teleproceso con varios } \\
\text { protocolos de comunicación. Es un sistema } \\
\text { abierto con interfaces de todo tipo al exterior. }\end{array}$ \\
\hline
\end{tabular}

Tabla 2. Ponderación de procesamiento distribuido.

\begin{tabular}{|c|l|}
\hline \multicolumn{2}{|c|}{ Procesamiento distribuído } \\
\hline Grado & \multicolumn{1}{|c|}{ Descripción } \\
\hline 0 & $\begin{array}{l}\text { El sistema no contribuye en la transferencia } \\
\text { de datos o funciones entre los procesadores } \\
\text { de la empresa. }\end{array}$ \\
\hline 1 & $\begin{array}{l}\text { El sistema prepara datos para el usuario final } \\
\text { en otro equipo de la empresa. }\end{array}$ \\
\hline 2 & $\begin{array}{l}\text { El sistema prepara datos para transferencia, } \\
\text { los transfiere y son procesados en otro equipo } \\
\text { de la organización. }\end{array}$ \\
\hline 3 & $\begin{array}{l}\text { El sistema ofrece un procesamiento distribuido } \\
\text { y la transferencia de datos es en línea, en una } \\
\text { dirección. }\end{array}$ \\
\hline 4 & $\begin{array}{l}\text { El sistema ofrece un procesamiento distribuido } \\
\text { y la transferencia de datos es en línea, en ambas } \\
\text { direcciones. }\end{array}$ \\
\hline 5 & $\begin{array}{l}\text { Las funciones de procesamiento son dinámi- } \\
\text { camente ejecutadas en el equipamiento más } \\
\text { adecuado. }\end{array}$ \\
\hline
\end{tabular}

Tabla 3. Ponderación de entrada on-line de datos.

\begin{tabular}{|c|l|}
\hline \multicolumn{2}{|c|}{ Entrada on-line de datos } \\
\hline Grado & \multicolumn{1}{|c|}{ Descripción } \\
\hline 0 & $\begin{array}{l}\text { Todas las transacciones son procesadas en } \\
\text { modo batch. }\end{array}$ \\
\hline 1 & $\begin{array}{l}\text { De 1\% al 7\% de las transacciones son entradas } \\
\text { de datos en línea. }\end{array}$ \\
\hline 2 & $\begin{array}{l}\text { De 8\% al 15\% de las transacciones son entradas } \\
\text { de datos en línea. }\end{array}$ \\
\hline 3 & $\begin{array}{l}\text { De 16\% al 23\% de las transacciones son } \\
\text { entradas de datos en línea. }\end{array}$ \\
\hline 4 & $\begin{array}{l}\text { De 24\% al 30\% de las transacciones son } \\
\text { entradas de datos en línea. }\end{array}$ \\
\hline 5 & $\begin{array}{l}\text { Más del 30\% de las transacciones son entradas } \\
\text { de datos en línea. }\end{array}$ \\
\hline
\end{tabular}

Tabla 4. Ponderación de diseño para la eficiencia de usuario final.

\begin{tabular}{|c|l|}
\hline \multicolumn{2}{|c|}{ Diseño para la eficiencia de usuario final } \\
\hline Grado & \multicolumn{1}{|c|}{ Descripción } \\
\hline 0 & No ofrece ningún aspecto de diseño funcional. \\
\hline 1 & $\begin{array}{l}\text { Ofrece de uno a tres aspectos de diseño } \\
\text { funcional. }\end{array}$ \\
\hline 2 & $\begin{array}{l}\text { Ofrece de uno a tres, cuatro a cinco aspectos } \\
\text { de diseño funcional. }\end{array}$ \\
\hline 3 & $\begin{array}{l}\text { Más de cinco aspectos de diseño funcional, } \\
\text { pero no hay requerimientos específicos del } \\
\text { usuario en cuanto a amigabilidad del sistema. }\end{array}$ \\
\hline 4 & $\begin{array}{l}\text { Más de cinco aspectos de diseño funcional, y } \\
\text { con requerimientos en cuanto a amigabilidad } \\
\text { del sistema suficientes para generar actividades } \\
\text { específicas incluyendo factores como minimi- } \\
\text { zación de la digitación. }\end{array}$ \\
\hline 5 & $\begin{array}{l}\text { Más de cinco aspectos de diseño funcional, y } \\
\text { con requerimientos en cuanto a la amigabili- } \\
\text { dad suficientes para utilizar herramientas } \\
\text { especiales y procesos especiales para demos- } \\
\text { trar anticipadamente que los objetivos fueron } \\
\text { alcanzados. }\end{array}$ \\
\hline
\end{tabular}

\section{Comparación de herramientas}

La fase de comparación pretende, como su nombre lo indica, establecer valoraciones de cada sistema ERP según su desempeño en los criterios ya mencionados, y el respectivo análisis de los valores obtenidos. 
Tabla 5. Ponderación de actualización on-line.

\begin{tabular}{|c|l|}
\hline \multicolumn{2}{|c|}{ Actualización on-line } \\
\hline Grado & \multicolumn{1}{c|}{ Descripción } \\
\hline 0 & No permite actualización en línea. \\
\hline 1 & $\begin{array}{l}\text { El sistema permite la actualización en línea de } \\
\text { uno a tres archivos lógicos internos. }\end{array}$ \\
\hline 2 & $\begin{array}{l}\text { El sistema permite la actualización en línea de } \\
\text { más de tres archivos lógicos internos. }\end{array}$ \\
\hline 3 & $\begin{array}{l}\text { El sistema permite la actualización en línea } \\
\text { de la mayoría de los archivos lógicos internos. }\end{array}$ \\
\hline 4 & $\begin{array}{l}\text { El sistema permite, además del ítem anterior, } \\
\text { la protección contra pérdidas de datos. }\end{array}$ \\
\hline 5 & $\begin{array}{l}\text { El sistema ofrece, además del ítem anterior, } \\
\text { procesos para automatizar la recuperación. }\end{array}$ \\
\hline
\end{tabular}

Tabla 6. Ponderación de puestos múltiples.

\begin{tabular}{|c|l|}
\hline \multicolumn{2}{|c|}{ Puestos múltiples } \\
\hline Grado & \multicolumn{1}{|c|}{ Descripción } \\
\hline 0 & $\begin{array}{l}\text { No se considera la instalación en más de un } \\
\text { equipo. }\end{array}$ \\
\hline 1 & $\begin{array}{l}\text { Se considera la instalación en múltiples equipos } \\
\text { operando sobre el mismo ambiente de hardware } \\
\text { y software. }\end{array}$ \\
\hline 2 & $\begin{array}{l}\text { Se considera la instalación en múltiples } \\
\text { equipos operando sobre ambientes similares } \\
\text { de hardware y software. }\end{array}$ \\
\hline 3 & $\begin{array}{l}\text { Se considera la instalación en múltiples } \\
\text { equipos operando sobre diferentes ambientes } \\
\text { de hardware y/o software. }\end{array}$ \\
\hline 4 & $\begin{array}{l}\text { Además de los ítems 1 y 2, se considera un plan } \\
\text { de mantenimiento y documentación. }\end{array}$ \\
\hline 5 & $\begin{array}{l}\text { Además del ítem 3, se considera un plan de } \\
\text { mantenimiento y documentación. }\end{array}$ \\
\hline
\end{tabular}

Tabla 7. Ponderación de facilidades de cambio.

\begin{tabular}{|c|c|}
\hline \multicolumn{2}{|c|}{ Facilidades de cambio } \\
\hline Grado & \multicolumn{1}{|c|}{ Descripción } \\
\hline 0 & No ofrece ninguno de los elementos descritos. \\
\hline 1 & Ofrece uno de los aspectos de facilidad de cambio. \\
\hline 2 & Ofrece dos de los aspectos de facilidad de cambio. \\
\hline 3 & Ofrece tres de los aspectos de facilidad de cambio. \\
\hline 4 & Ofrece cuatro de los aspectos de facilidad de cambio. \\
\hline 5 & Ofrece todos los aspectos de facilidad de cambio. \\
\hline
\end{tabular}

Debido a la complejidad que requiere establecer una medición apropiada de los criterios mencionados para cada sistema ERP, se incurre en la necesidad de realizar una agrupación de las características de cada software por elementos comunes, previo a la ponderación.

Teniendo en cuenta lo anterior, la evaluación de las herramientas se lleva a efecto por medio del siguiente protocolo:

1) Identificar elementos comunes de comparación.

2) Medir la ponderación de cada criterio en los elementos comunes identificados de las diferentes herramientas.

3) Comparar los resultados de la ponderación de cada criterio.

\section{Selección de módulos}

La fase final de la comparación la constituye la selección de los módulos que debe contener un sistema ERP orientado a logística portuaria, y deben corresponder a las diferentes etapas de funcionamiento de un software orientado a logística portuaria.

La identificación de los módulos se hace con base en el análisis de la funcionalidad de los elementos comunes que fueron medidos usando los criterios de comparación.

La medición promedio de los elementos comunes en los diferentes sistemas es usada como criterio para medir la funcionalidad que tiene dicho elemento y, por tanto, incluirlo en el conjunto de módulos seleccionados.

\section{RESULTADOS DE IMPLEMENTACIÓN DE LA METODOLOGÍA DE COMPARACIÓN DE SISTEMAS ERP ORIENTADOS A LOGÍSTICA PORTUARIA}

En la sección de resultados se implementa la metodología propuesta para la comparación de tres sistemas ERP orientados a logística portuaria y se discuten los hallazgos.

\section{Búsqueda de herramientas ERP y definición de métricas de comparación \\ Como punto inicial identificaremos algunos sistemas de planificación de recursos empresariales,}


específicamente orientados a la logística portuaria, entre ellos en el ámbito latinoamericano se destaca la presencia de tres: $\mathrm{Sinco}^{4}$ (Sistema usado en puertos mexicanos), SilogPort ${ }^{5}$ (Sistema usado en el puerto de Valparaiso, Chile); y se incluyó, además, el sistema Transkal ${ }^{6}$ (Sistema usado en puertos de España).

En la Tabla 8 se presenta la comparación de las características generales de cada uno de los sistemas seleccionados; un análisis superficial refleja un alto grado de automatización en elementos de cálculo y validación, además de contar con la integración de políticas vigentes. En todos los casos se posee un diseño modular, incluso con arquitectura abierta. Asimismo, se destaca la multiplicidad de canales para su uso, entre otros.

Anteriormente fueron expuestos tanto los criterios que serán usados para la comparación de sistemas ERP orientados a logística portuaria, como sus niveles de calificación. En este sentido se definieron siete factores que abordan características funcionales del sistema y aplican de manera coherente en la medición de sistemas de este tipo; los factores seleccionados son: Comunicación de datos, Procesamiento distribuido, Entrada on-line de datos, Diseño para la eficiencia de usuario final, Actualización on-line, Puestos múltiples, Facilidad de cambio.

\section{Comparación de herramientas}

Para la comparación de resultados se seguirán las tres etapas del protocolo expuesto:

\section{Identificación de elementos comunes de comparación:} Un análisis preliminar de las características expuestas en la Tabla 8, permite identificar una serie de elementos comunes en los sistemas ERP para logística portuaria seleccionados.

La lista de elementos comunes es presentada en la Tabla 9; asimismo, se señala en cuales sistemas está presente.

Del análisis inicial de la Tabla 9, es posible afirmar que el sistema Transkal es el más completo,

\footnotetext{
4 http://www.si-ti.com.mx/index.php

5 https://www.silogport.cl/web/silogport/inicio

6 http://www.transkal.com/es/soluciones_informaticas/detalle. php?cat=presentacion_es\&modulo $=4$
}

en términos de granularidad de los elementos. No obstante, Transkal comparte funcionalidad con muchos de los elementos identificados en Sinco. Asimismo, que el sistema SILOGPORT se orienta más a labores de transporte y movilidad.

Medición de cada criterio en los elementos comunes identificados de las diferentes herramientas: $\mathrm{La}$ medición de cada factor para los elementos comunes identificados se presenta por medio de la realización de figuras para cada factor.

Las Figuras 3 al 9, respectivamente, representan la mediación de cada uno de los siete factores señalados como criterios funcionales. En todas las figuras el sistema Transkal es presentado en tonalidad verde, Sinco corresponde a la línea roja y Silogport la línea gris.

Resultados comunicación de datos: En relación al factor de comunicación de datos se observa que las herramientas Sinco y Transkal presentan una mayor funcionalidad en el nivel de transmisión de información en los diferentes módulos analizados principlamente los de control de carga y almacénes, facturación, coordinación de operaciones, monitoreo, planeación y generación de consulta; en donde los sistemas ofrecen teleprocesos con varios protocolos de comunicación (ver Figura 3).

Resultados procesamiento distribuído: En la Figura 4, se observa el factor de procesamiento distribuido evidenciándose claramente que la herramienta con mayor funcionalidad sobre la efectividad, accesibilidad y complejidad del sistema es el Transkal presentando estabilidad en el 100\% de los módulos analizados.

Resultados entrada on line de datos: Respecto al factor que cuantifica la entrada de datos en línea prevista para el sistema se observa que existe un comportamiento estable en la funcionalidad de las herramientas Transkal y Sinco; pero en los módulos relacionados con aduana, gestión de flota, logística de almacén y transporte los tres ERP analizados presentan baja funcionalidad en este tema (ver Figura 5).

Resultados diseño para la eficiencia del usuario final: La Figura 6 muestra la comparación de las herramientas respecto al factor relacionado con el 
Tabla 8. Identificación de tres sistemas ERP orientados a logística portuaria.

\begin{tabular}{|c|c|c|c|}
\hline ERP & SINCO & 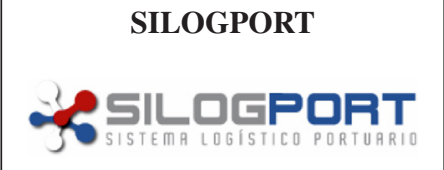 & 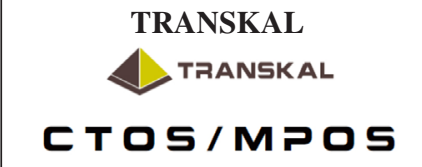 \\
\hline 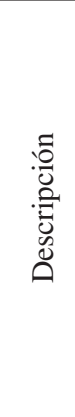 & $\begin{array}{l}\text { Es un completo sistema integral de } \\
\text { información, diseñado especialmente } \\
\text { para las necesidades de control y } \\
\text { seguridad en las operaciones de una } \\
\text { administración portuaria integral, } \\
\text { así como sus intercambios de } \\
\text { información con las dependencias } \\
\text { rectoras a nivel federal y usuarios } \\
\text { externos por medio de internet. }\end{array}$ & $\begin{array}{l}\text { Se destaca por ser el primer } \\
\text { Port Community System de } \\
\text { Latinoamérica y como tal viene } \\
\text { a instalar una nueva forma de } \\
\text { gestionar los negocios del mundo } \\
\text { marítimo portuario. Además, cuenta } \\
\text { con el apoyo principal de Puerto de } \\
\text { Valparaíso. }\end{array}$ & $\begin{array}{l}\text { Es una solución informatizada } \\
\text { y completa aplicable a las } \\
\text { empresas relacionadas con las } \\
\text { tareas propias de una terminal } \\
\text { portuario. Comprende la gestión de } \\
\text { operaciones (buque, tren y tierra), } \\
\text { gestión de recursos (materiales y } \\
\text { humanos), tanto propios como } \\
\text { ajenos, Almacenaje de partidas, } \\
\text { Graneles, Líquidos, Gestión de } \\
\text { terminal de contenedores. }\end{array}$ \\
\hline 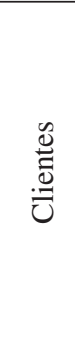 & $\begin{array}{l}\text { Los clientes de Sinco en su mayoría } \\
\text { se encuentran distribuidos en la } \\
\text { ciudad de México, algunos de estos } \\
\text { clientes son: } \\
\text { - Apiver } \\
\text { - Api2boca } \\
\text { - Aapitan Apialta }\end{array}$ & $\begin{array}{l}\text { Los clientes de SilogPort se centran } \\
\text { en su gran mayoría en la ciudad } \\
\text { de Santiago pero cuentan con } \\
\text { otros puntos a nivel mundial como } \\
\text { Colombia, Perú y Argentina, algunos } \\
\text { de ellos son } \\
\text { - Unipack } \\
\text { - Transfresh } \\
\text { - Schindler }\end{array}$ & $\begin{array}{l}\text { Los principales clientes de transkal } \\
\text { se encuentran ubicados en España } \\
\text { y el país Vasco, de los cuales se } \\
\text { destacan: } \\
\text { - Fletes Logisticos } \\
\text { - Marbeco, S.A. } \\
\text { - Portsur Castellón } \\
\text { - Consulmar }\end{array}$ \\
\hline 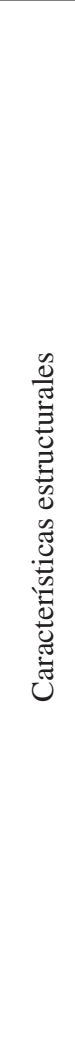 & $\begin{array}{l}\text { 1) Diseño modular completamente } \\
\text { integral que provee seguridad } \\
\text { e integridad de la información } \\
\text { (Control) } \\
\text { 2) Sistema que se paramétriza } \\
\text { para realizar de manera } \\
\text { automática los cálculos y } \\
\text { validar que se apliquen las } \\
\text { tarifas y políticas vigentes } \\
\text { (brindando consistencia y } \\
\text { seguridad). } \\
\text { 3) } \\
\text { infte ma in te gral, con } \\
\text { todo el sistema al instante en } \\
\text { que los datos son generados } \\
\text { o capturados (información en } \\
\text { línea). } \\
\text { Información protegida en bases } \\
\text { de datos (evitando accesos por } \\
\text { fuera del sistema) y a nivel } \\
\text { aplicaciones, los datos solo } \\
\text { son accedidos por los usuarios } \\
\text { con los privilegios necesarios } \\
\text { para ello. }\end{array}$ & $\begin{array}{l}\text { 1) Permite contar oportunamente } \\
\text { con la de cada eslabón de la } \\
\text { cadena logístico-portuaria, } \\
\text { ofreciendo visibilidad y } \\
\text { trazabilidad del flujo de cargas, } \\
\text { tanto fuera como dentro del } \\
\text { puerto. } \\
\text { 2) Permite anticipar la demanda de } \\
\text { servicios logísticos y portuarios } \\
\text { en los principales nodos de la } \\
\text { cadena y con ello planificar } \\
\text { la oferta y disponibilidad de } \\
\text { recursos, dando mayor fluidez } \\
\text { al traslado físico de las cargas } \\
\text { y optimizando el uso de la } \\
\text { infraestructura instalada. } \\
\text { Permite sincronizar el traslado } \\
\text { físico de las cargas con su } \\
\text { tramitación documental, de } \\
\text { modo de asegurar un flujo } \\
\text { expedito al interior de las } \\
\text { instalaciones portuarias. } \\
\text { Reduce la cantidad de trámites } \\
\text { manuales y presenciales, así } \\
\text { como la redigitación de datos. }\end{array}$ & $\begin{array}{l}\text { 1) Tecnología abierta evolutiva } \\
\text { basada en herramientas } \\
\text { software estándar. } \\
\text { 2) } \\
\text { Multiempresa, multicentro, } \\
\text { multiusuario, multimoneda } \\
\text { y capacidad multilingüe. } \\
\text { 3) Dato único interactuando en } \\
\text { tiempo real. } \\
\text { 4) Amplitud funcional e inte- } \\
\text { gración de los procesos del } \\
\text { negocio. } \\
\text { 5) Sistema accesible vía internet. } \\
\text { 6) Multiconcurrencia en la } \\
\text { ejecución de funciones. }\end{array}$ \\
\hline
\end{tabular}


Tabla 9. Elementos comunes en los tres sistemas ERP para logística portuaria seleccionados.

\begin{tabular}{|c|c|c|c|}
\hline Elementos comunes & $\begin{array}{l}0 \\
\text { Z } \\
\text { Zn }\end{array}$ & 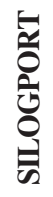 & 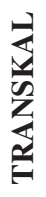 \\
\hline Contabilidad & $\mathrm{X}$ & $X$ & $\mathrm{X}$ \\
\hline Control de buques & $\mathrm{X}$ & $\mathrm{X}$ & $\mathrm{X}$ \\
\hline Control de carga y almacenes & $\mathrm{X}$ & $X$ & $\mathrm{X}$ \\
\hline Facturación & $\mathrm{X}$ & $\mathrm{X}$ & $\mathrm{X}$ \\
\hline Administración & & $\mathrm{X}$ & $\mathrm{X}$ \\
\hline Coordinación de operaciones & & $\mathrm{X}$ & $\mathrm{X}$ \\
\hline Monitoreo & & $\mathrm{X}$ & $\mathrm{X}$ \\
\hline Planificación & & $X$ & $\mathrm{X}$ \\
\hline Generador consulta & & $\mathrm{X}$ & $\mathrm{X}$ \\
\hline Gestión de carga & & $X$ & $\mathrm{X}$ \\
\hline Aduana & & & $\mathrm{X}$ \\
\hline Gestión de flota & & & $\mathrm{X}$ \\
\hline Logística de almacén & & & $\mathrm{X}$ \\
\hline Logística de transporte & & & $\mathrm{X}$ \\
\hline
\end{tabular}

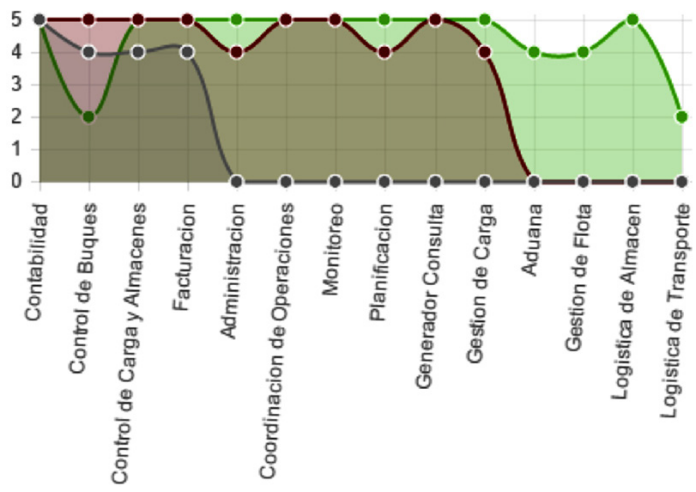

Figura 3. Resultados comunicación de datos.

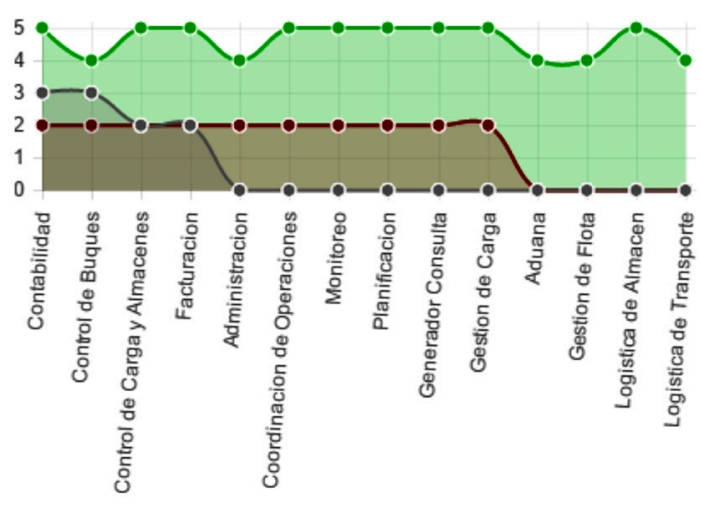

Figura 4. Resultados procesamiento distribuido.

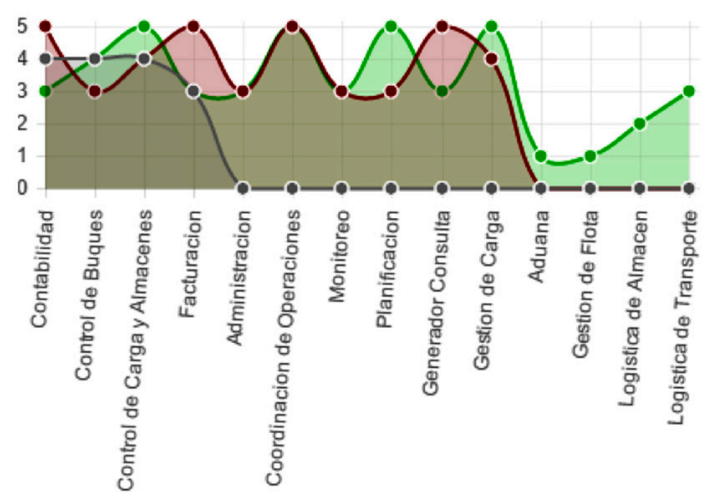

Figura 5. Resultados entrada on-line de datos.

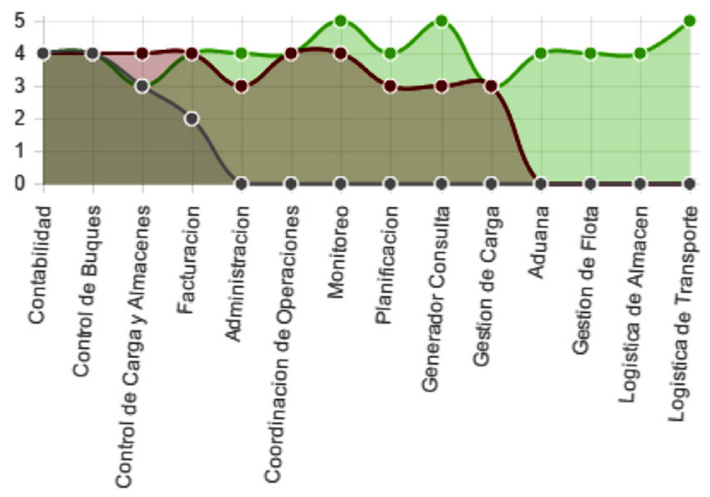

Figura 6. Resultados diseño para la eficiencia del usuario final.

diseño para la eficiencia de usuario final mostrando que el ERP con mayor funcionalidad es el Transkal principalmente en los módulos de monitoreo, planificación, generador de consulta, gestión de flota, logística de almacén y transporte.

Resultados actualización on-line: El factor de actualización de datos de las herramientas hace referencia a la posibilidad de actualización en linea de archivos lógicos observando que el ERP Sinco muestra una mayor funcionalidad en el $57,1 \%$ de los módulos analizados; mientras que el Transkal presenta mayor funcionalidad en los módulos de aduana, gestión de flota, logística de almacén y transporte (ver Figura 7).

Resultados puestos múltiples: En la Figura 8, se observa claramente que el ERP Transkal presenta a lo largo de los módulos analizados una mayor funcionalidad respecto a la posibilidad de poder 


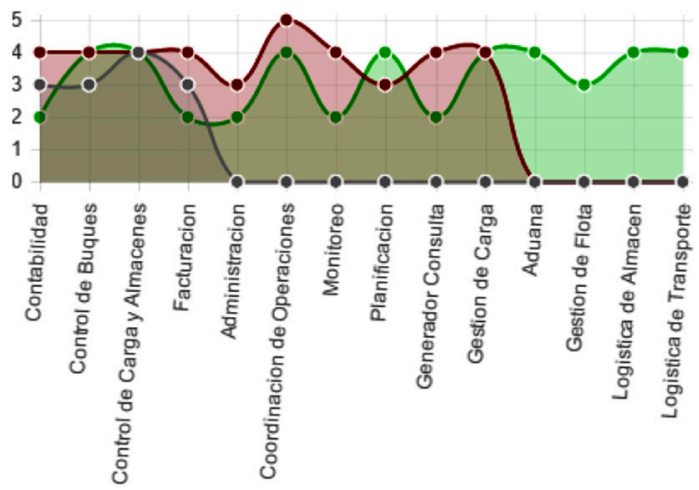

Figura 7. Resultados actualización on-line.

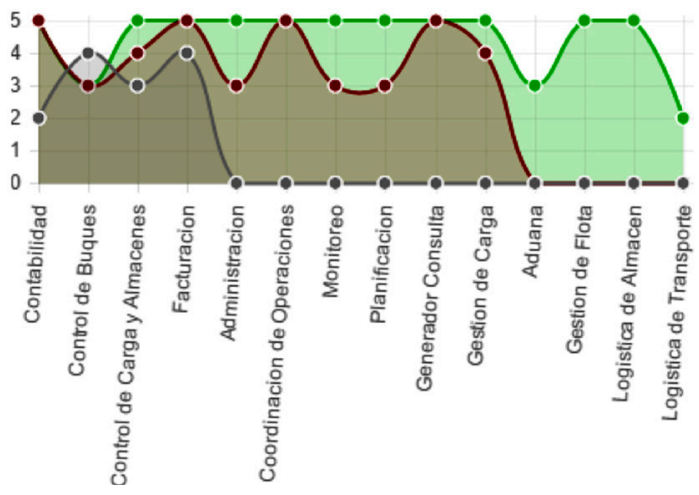

Figura 8. Resultados puestos múltiples.

instalar la herramienta en multiples equipos al interior de una organización o entre varias organizaciones.

Resultados Facilidad de Cambio: Las posibilidades que muestran las herramientas Transkal y Sinco

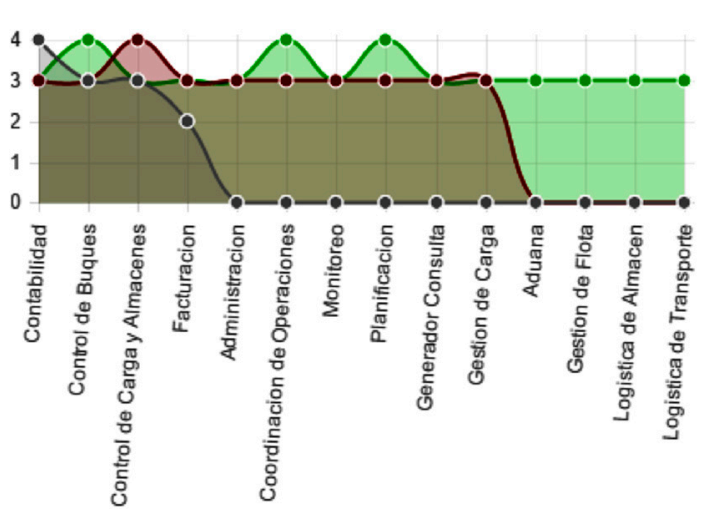

Figura 9. Resultados facilidad de cambio. en cuanto a su facilidad de cambio para su mantenimiento tienen un comportamiento en su funcionalidad moderado en los módulos analizados (ver Figura 9).

\section{Comparación de los resultados de la ponderación de cada criterio}

La comparación realizada a cada ERP seleccionado sobre los resultados de los siete factores se puede determinar que de las tres herramientas las que cuentan con mayor funcionalidad es Transkal resaltando los elementos de comunicación de datos, el procesamiento distribuido, seguido de la eficiencia de usuario final, puestos múltiples y facilidad de cambio.

Se observó que el ERP Sinco a pesar de contar con buena funcionalidad en los elementos analizados en los módulos relacionados con aduana, gestión de flota, logistica de almacén y transporte presentan mediciones bajas en todos los factores.

Por su parte, la herramienta Silogport presenta un comportamiento estable en su funcionalidad en los módulos relacionados con contabilidad, control de buques, control de carga y almacénes. Pero que se carece de parámetros para establecer la medición de otros factores como administración, cordinación de operaciones, monitoreo, planificación, generador de consulta, gestión de carga, aduana, gestión de flota, logística de almacén y transporte, toda vez que no cuenta con tales elementos.

\section{Selección de módulos}

En secciones previas, basados en la ponderación de diferentes sistemas ERP, se establecieron los

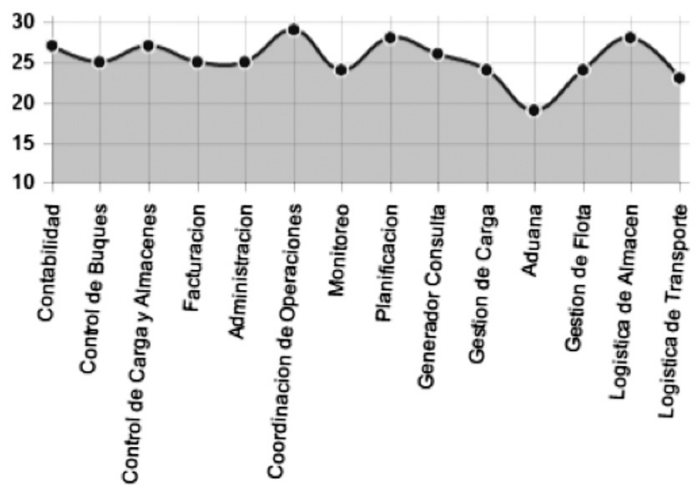

Figura 10. Resultados promedio por elementos comunes. 
elementos necesarios para la comparación de la funcionalidad de los diferentes sistemas.

La Figura 10 muestra el promedio de ponderaciones obtenidas para los diferentes elementos comunes identificados. Se resalta alta ponderación de los elementos Control de carga y almacénes, Coordinación de operaciones, Planificación, Generador de consulta, Gestión de flota, Logística de almacén y de transporte, lo que se considera un indicio de la necesidad de incluir tales elementos como características funcionales de un sistema ERP orientado a la logística portuaria.

Asimismo, elementos como Control de buques, Contabilidad, Aduanas, Facturación y administración, se considera oportuna su inclusión, en la medida que responden a diferentes etapas de funcionamiento de un sistema de este tipo.

Tras haber analizado, establecido una comparación de las similitudes de cada ERP y las particularidades de cada elemento común, se seleccionaron y nombraron los módulos más pertinentes para hacer parte necesaria de un sistema ERP para logística portuaria, los que se presentan en Tabla 10. Los doce módulos seleccionados comprenden cada una de las etapas de funcionamiento de un software orientado a logística portuaria.

Es necesario aclarar que los módulos seleccionados como guía para la elaboración de un sistema ERP orientado a logística portuaria, incluyen componentes de información básica que tanto los agentes del sistema y los usuarios en general deben conocer, asimismo, las variables de ejecución de este; no obstante, se descartaron algunos módulos importantes, toda vez que no se adaptan a las necesidades que se requieren en el sistema.

Tabla 10. Resultado de módulos seleccionados.

\begin{tabular}{|l|l|}
\hline \multicolumn{2}{|c|}{ Módulos seleccionados } \\
\hline Aduanas & Control de carga y almacenes \\
\hline Logística de almacén & Facturación \\
\hline Administración & Coordinación de operaciones \\
\hline Control de buques & Generador de consulta \\
\hline Gestión de flota & Planificador \\
\hline Contabilidad & Logística de transporte \\
\hline
\end{tabular}

\section{CONCLUSIONES}

El desarrollo de un sistema ERP representa para la empresa una gran inversión no solo económica sino también de otros recursos, como es el tiempo y esfuerzo de sus empleados, y la migración de información de un sistema a otro con los riesgos que este proceso implica. Se espera que un sistema ERP una vez implementado dure unos cuantos años y acompañe a la empresa en sus proyectos, planes y objetivos de negocio. Es por esto, que la adecuada selección de los módulos que deben componer el sistema ERP es una tarea delicada.

Es importante poder seleccionar los módulos que mejor se adecuen a las necesidades de la empresa en varios aspectos, no solo los económicos sino funcionales, estratégicos, y técnicos inherentes a su servicio. Es importante también que el ERP no quede obsoleto al poco tiempo de implementación, pero tampoco que sea tan complejo para la organización que no sea aprovechada totalmente su funcionalidad. Todos estos puntos hacen pensar que esta etapa es un proceso crítico. Más aún, si se tiene en cuenta que la selección de sistemas ERP no es un área de mucho estudio; son pocas las metodologías que guíen a los directivos y miembros del equipo de proyecto en el trabajo de seleccionar los módulos que deben componer la herramienta ERP.

En este artículo se propuso una metodología orientada a la comparación de sistemas de recursos empresariales de planificación para logística portuaria como apoyo a la selección de los módulos prioritarios en su implementación. Como tarea inicial se identificaron algunos sistemas ERP, específicamente orientados a la gestión logística, en el ámbito latinoamericano e iberoamericano; definiendo para la ejecución de esta tarea tres herramientas tipo ERP logísticos (Transkal, Sinco y Silogport) como casos de comparación de plataformas TIC, asimismo, se establecieron criterios de evaluación de tales herramientas y se realizó la evaluación de cada módulo que componen los casos seleccionados.

La metodología establecida para la comparación de los sistemas ERP orientados a logística portuaria se basó en la valoración de los criterios, el análisis de los parámetros obtenidos y la selección de los módulos que se consideran básicos como parte de sistema de este tipo. Los criterios recogen aspectos 
funcionales de los sistemas, que tradicionalmente no son tenidos en cuenta a la hora de realizar una comparación, o en las metodologías de selección de ERP.

La validación de la metodología por medio de la comparación de tres sistemas ERP para logística portuaria permitió la identificación de doce módulos que responden a las necesidades de un sistema ERP orientado a logística portuaria.

La metodología expuesta apunta a cubrir todo el ciclo de selección de módulos de un ERP y ser los más flexible posible, adecuándose a implementaciones grandes y chicas. Asimismo, ofrece un espacio para apoyar la toma de decisiones empresariales basado en la rigurosidad académica que da la elaboración de metodologías propias para el desarrollo de procesos.

Finalmente, el trabajo desarrollado ofrece campos abiertos para posibles investigaciones: se pueden incorporar estudios para valorar costos $v s$. beneficios de desarrollar un sistema a medida o implementar una solución comercial basado en aspectos funcionales, estratégicos y técnicos; se puede valorar a profundidad, para necesidades específicas, la definición de criterios de elección definidos en el paso 4.

\section{REFERENCIAS}

[1] V. Kumar, B. Maheshwari and U. Kumar. "Enterprise resource planning systems adoption process: a survey of Canadian organizations". International Journal of Production Research 2002. Vol. 40, pp. 509523. 2002.

[2] K. Olsen and P. Sætre. "ERP for SMEsis proprietary software an alternative?". Business Process Management Journal. Vol. $13 \mathrm{~N}^{\circ}$ 3, pp. 379-389. 2007.

[3] C. Stefanou. "The selection process of enterprise resource planning (ERP) systems". in Americas Conference on Information Systems. 2000.

[4] F. Wilson, J. Desmond and H. Roberts. "Success and failure of MRPII implementation". British Journal of Management. Vol. 5, pp. 221-240. 1994.

[5] D. Hicks and K. Stecke. "The ERP maze: enterprise resource planning and other production and inventory control software". IIE Solutions. Vol. 27 No 8, pp. 12-16. 1995.

[6] A. Teltumbde. "A framework of evaluating ERP projects". International Journal of Production Research. Vol. 28, pp. 4507-4520. 2000.

[7] C.-C. Wei and M.-J. Wang. "A comprehensive framework for selecting an ERP system". International Journal of Project Management. Vol. 22, pp. 161-169. 2004.

[8] M. Haddara. "ERP Selection: The SMART Way". Procedia Technology. Vol. 16, pp. 394403. 2014.

[9] Y. Su and C. Yang. "A structural equation model for analyzing the impact of ERP on SCM". Expert Systems with Applications. Vol. 37, pp. 456-469. 2010.

[10] K. Kumar and J. Hillegersberg. "Enterprise resource planning: Introduction". Communications of the ACM. Vol. $43 \mathrm{~N}^{\circ} 4$, pp. 22-26. 2000.

[11] D. Reuther and G. Chattopadhyay. "Critical factors for enterprise resources planning system selection and implementation projects within small to medium enterprises". In Reuther D, Chattopadhyay G, editors. Critical Factors for Enterprise Resources Planning System Selection and Implementation Projects within Small to Medium Enterprises. Engineering Management Conference. 2004.

[12] P. Sudhaman and C. Thangavel. "Efficiency analysis of ERP projects-software quality perspective". International Journal of Project Management. Vol. 33, p. 961-970. 2015.

[13] M. Haddara and A. Elragal. "ERP Lifecycle: A Retirement Case Study". Information Resources Management Journal. Vol. 26 No 1, pp. 1-11. 2012.

[14] D. Aloini, R. Dulmin and V. Mininno. "Risk assessment in ERP projects". Information Systems. Vol. 37 No 3, pp. 183-199. 2012.

[15] Y. van Everdingen, J. Hillegersberg and E. Waarts. "Enterprise resource planning: ERP adoption by European midsize companies". Communication of the Association for Computing Machinery. Vol. $43 \mathrm{~N}^{\mathrm{o}} 4$, pp. 27-31. 2000.

[16] S. Rao. "Enterprise resource planning: business needs and technologies". Industrial Management and Data Systems. Vol. 100, pp. 81-88. 2000. 
[17] A. Winkelmann. "Experiences While Selecting, Adapting and Implementing ERP Systems in SMEs: A Case Study". in Proceedings of the Americas Conference on Information Systems. 2008.

[18] H. Sun, W. Ni and R. Lam. "A step-by-step performance assessment and improvement method for ERP implementation: Action case studies in Chinese companies". Computers in Industry. Vol. 68, p. 40-52. 2015.

[19] S. Hung, S. Chang and P. Lee. "Critical Factors of ERP Adoption for Small- and Medium-Sized Enterprises: An Empirical Study". In Proceedings of the Pacific Asia Conference on Information Systems. 2004.

[20] S. Perçin. "Using the ANP approach in selecting and benchmarking ERP system". Benchmarking: An International Journal. Vol. $15 \mathrm{~N}^{\circ}$ 5, pp. 630-649. 2008.

[21] C. Wei, C. Chien and M. Wang. "An AHPbased approach to ERP system selection". Int. J. Production Economics. Vol. 96, pp. 47-62. 2005.

[22] M. Argyropoulou, G. Ioannou, K. Soderquist and J. Motwani. "Managing ERP system evaluation and selection in SMEs using the six-imperatives methodology". International Journal of Procurement Management. Vol. 1 $\mathrm{N}^{\circ}$ 4, pp. 430-452. 2008.
[23] J. Verville and A. Halingten. "A six-stage model of the buying process for ERP software". Industrial Marketing Management. Vol. 32, pp. 585-594. 2003.

[24] P. Tan, S. Lee and A. Goh. "Multi-criteria decision techniques for context-aware B2B collaboration in supply chains". Decision Support Systems. Vol. $52 \mathrm{~N}^{\mathrm{o}}$ 4, pp. 779789. 2012.

[25] A. Hakim and H. Hakim. "A practical model on controlling the ERP implementation risks". Information Systems. Vol. 35, pp. 204-214. 2010.

[26] M. Ziaee, M. Fathian and S. Sadjadi. "A modular approach to ERP systemselection: a case study". Information Management \& Computer Security. Vol. $14 \mathrm{~N}^{\circ}$ 5, pp. 485495. 2006.

[27] H. Kilic, S. Zaim and D. Delen. "Development of a hybrid methodology for ERP system selection:". Decision Support Systems. Vol. 66, pp. 82-92. 2014.

[28] H. Kilic, S. Zaim and D. Delen. "Selecting "The Best" ERP system for SMEs using a combination of ANP and PROMETHEE methods". Expert Systems with Applications. Vol. 42, pp. 2343-2352. 2015.

[29] J.A. McCall, P. G. Richards and G. F. Walters. "Factors in Software Quality". 1977. 